\title{
Hipercalciuria idiopática en pediatría. Experiencia de 5 años
}

\author{
Francisco Cano Sch. ${ }^{\text {; }}$ Eugenio Rodríguez S. ; M. Angela Delucchi B.1 \\ Eduardo Wolff P. ${ }^{\prime}$; Angel Fuentes Z. ${ }^{2}$; Hilda Kunzagk S. ${ }^{3}$
}

\section{Idiopathic hypercalciuria}

\begin{abstract}
local reference volues are imporfant for the correct approach to the diagnosis and management of idiopathic hypercaleiuria. Ninety seven healthy children were evaluated to determine daily narmal urine calciurn excretion. A meon value of $0.13 \mathrm{M}=0.081$, was obtained for the colcium/creatinine urinary index. We also studied the relationship between urinory caltium and sodium excretion in $=47$ ). A posilive, stolistically significant relation could be stablished tp $<0.011$. To examine the relationship belween colcium creatinine urinary index and calcium excretion in a 24 hour urine sample, 44 children whith hypercalciuria were evaluated. A strong correlatlon $(r-0.89)$ was oblained showing the close associalion among results from both methods, $p<0.001$. Two lest were evalualed in seventy eighl potients with hypercalciurta in order to identity renal and absortive hypercalciuria subtypes. A one week calcium restriction test showed less non diagnosis results $[0 / 4 S$ cases) than the dossic calcium loadig lesi $16 / 33$ casest, leading to better classification of these patients.
\end{abstract}

(Key words: calcium metabolism disorders, idiopathic hypercalciuria, urine colcium/crealinine Index.)

La hipercalciuria idiopática ha sido objeto de creciente interés en los últimos años al confirmarse su asociación etiológica con la hematuria y la litiasis de vía urinaria, en frecuencias que oscilan entre 25 a $40 \% 1,2$ y 40 a $75 \%$ respectivamente ${ }^{3}$. Esta relación, de evidente significado terapéutico, ha permitido un enfoque más racional en el manejo de estas afecciones, que representan un alto porcentaje de la consulta en una policlínica de nefrourología. En 10 a $25 \%$ de pacientes hematúricos con hipercalciuria idiopática se ha evidenciado la posterior aparición de litiasis urinaria ${ }^{1,4}$, como hemos comprobado en tres de cincuenta pacientes atendidos en nuestra unidad en tres anos ${ }^{2}$. Dicha asociación se ha observado con mayor frecuencia en pacientes con hematuria macroscópica e historia familiar de litiasis.

1. Unidad de Nefrologia y Hemodiślisis, Universidad de Chile, Departamento de Pediatria, Area Oriente, Hospital Luis Calvo Mackenna.

2. Unidad de Lactantes, Hospital Luis Calvo Mackenna.

3. Laboratorio de Bioquímica, Hospital Luis Calvo Mackenna.
El estudio de la hipercalciuria idiopática es relativamente nuevo en nuestro país, efectuándose sistemáticamente en pediatría desde hace aproximadamente 5 años a la fecha. Después de confirmar dicho diagnóstico en más de cien pacientes nos hemos planteado algunas interrogantes sobre los datos utilizados para identificar el trastomo, pues, ellos provienen de mediciones efectuadas en poblaciones de otros países. Tal es el caso de la excreción de calcio urinario, que en la literatura foránea fluctúa entre 2 y $8 \mathrm{mg} \cdot \mathrm{kg}$ · día como cifras máximas, aceptándose $4 \mathrm{mg} \cdot \mathrm{kg}$ en la mayoría de las publicaciones ${ }^{1-8}$. Otra duda dice relación con la posibilidad de obtener, en nuestro medio, buena correlación entre el contenido de calcio en orina de 24 horas y el índice calcio/ creatinina en una muestra aislada de orina obtenida en ayunas, para lo cual el índice correspondiente a una excreción de calcio de $4 \mathrm{mg} \cdot \mathrm{kg}$ - día seria $0,21^{8}$. También parece necesario aclarar si existe realmente una relación entre la excreción urinaria de sodio y la de calcio, cuya confirmaciớn es de notable interés dadas las implicancias terapéticas que reviste ${ }^{2} .9$. Finalmente parece importante identificar la prueba más adecuada 
para clasificar la hipercalciuria en los subtipos absortivo y renal, ya que el ensayo clásico de restricción y sobrecarga de calcio adolece dc ciertas limilaciones, especialmente alto porcentaje de resultados que no permiten hacer efectiva csta subdivisión, hasta $30 \%$ del total de estudios en una población hipercalciúrica. Actualmente se usa con mayor frecuencia una prucba de resuricción simple de calcio y sodio ${ }^{2,11}$, de acuerdo con cuyos resultados se clasifican los pacientes en que se normaliza la calciuria como hipercalciuria idiopática (Hi) absortiva, en tanto que aquellos en que persiste la excreción anormal como un subtipo renal de Hi. Esta experiencia se rcalizó con el propósito aportar información sobre la excreción urinaria de calcio y sodio, así como del índice sodio/creatinina en ninos sanos y en pacientes con hipercalciuria idiopática.

\section{Material y Método}

Se evaluaron 97 niños sctualmente sanos, de 3 a 14 años de edad, 52 varones. La mayoría eran pacientes que presentaron un episodio de infección urinaria hace más de 2 años. en los cuales se descartó la existencia de nefropalías o malformaciones de la vía urinaria. Seguidos con exámenes de orina por el ticmpo señalado, éste fue negativo en forma permanente. No sc hizo ningún intcnto por manipular la ingesta dictética en el períudo del estudio. De cada uno se obcuvo una muestra de orina en ayunas para medir el findice calcio/ereatinina, lo que fue hecho en el laboratorio de bioquírica del Hospital Luis Calvo Mackenna. En 47 de cstos niños se midió en forma concomitante la excreción utinaria de sodio.

Se cvaluaron además 78 pacientes entre 3 y 14 años, 48 varones. portadores de hipercalciuria idiopática descubierta a raíz de hematuria o lítiasis en un período de 3 años de seguimicnto. En 44 de ellos, junto con medir el índice $\mathrm{Ca} /$ creatinina, se recolectó orina de 24 horas para determinar la calciuria y establecer la relación existente cntre los resultados obtenidos con ambos procedimientos. Treinta y tres de los 78 pacientes hipercalciúricos fueron sometidos a prucba de restricción y sobrecarga de calcio (restricción y sobrecarga), para lo cual se aplico el estudio descrito en detalle en comunicaciones previas ${ }^{7 .}$ y cuarenta y cinco a la prueba dc restriccion simple de calcio y sodio (restricción simple). Esta última consiste en hacer una medición basal del índice calciuria/creatininuria en ayunas, scguida de una scmana de dieta restringida en calcio ( $250 \mathrm{mg} /$ dia) y sodio $(2 \mathrm{~g} / \mathrm{dia} a)$, para repetir la medición de calcio y creatinúna cn una muestra aislada de orina oblenida en ayunas al octavo día del estudio. Fodos los pacientes en quienes disminuýs el valor del índice cn la segunda muestra a menos de 0,21 fueron cutalogados como Hi absortiva, en los que se mantuvo sobre el valor indicado se diagnostico $\mathrm{Hi}$ renal. El esudio estadística incluý análisis de correlación simple entre las variables determinadas, con la finalidad de cncontrar asociaciones estadísticamente significativas $(p<0,05)$.

\section{Resultados}

En 97 pacientes sanos se obtuvo una excreción promedio de calcio en orina, medida a través del indice calciuria/creatininuria, de 0,13 (mediana 0,08 ). En los 47 niños en que se midió la excreción urinaria de sodio concomitantemente al índice $\mathrm{Ca} /$ creatinina, la natriuria fue $122 \mathrm{mg} \%$ (promedio), demostrándose correlación positiva entre ambos valores $(\mathbf{r}=0,4 \mathbf{l} ; \mathrm{p}<0,01)$ (figura 1 ). La medición simultánca de calciuria en orina de 24 horas en 44 de los 78 niños hipercalciúricos estudiados $(4,37 \pm 3,5$, mediana $4,2 \mathrm{mg})$ mostró una correlación altamente significativa $(r=0,89 ; p<$ 0,001 ) entre ambos métodos, permitiendo de este modo formular una ecuación para la curva, donde la excreción urinaria de calcio en 24 horas es igual a $0,87+17,74$ (Ca/crearinina) en muestra aislada de orina (figura 2 ).

En la evaluación de los 78 pacientes portadores de $\mathrm{Hi}$, considerados en conjunto, $58 \%$ respondieron de acuerdo al subtipo absortivo, $35 \%$ como en la forma renal y $6(7 \%)$ de un modo que no permitió clasiticarlos con los ensayos aplicados, que en todos estos últimos casos lue la prueba de restricción y sobrecarga. La frecuencia de resultados no conducentes a diagnóstico, usando exclusivamente cl ensayo de restricción y sobrecarga de calcio, había sido $25 \%$ en una publicación previa ${ }^{10}$. Al introducir -en cstc estudio- la

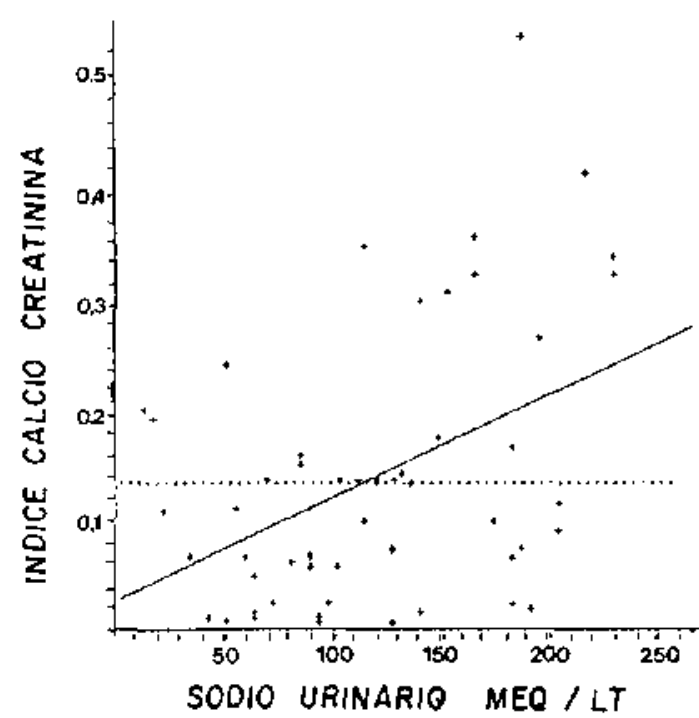

Figura 1: Relación entre excreción urinatia del calcio vs. sodio. 


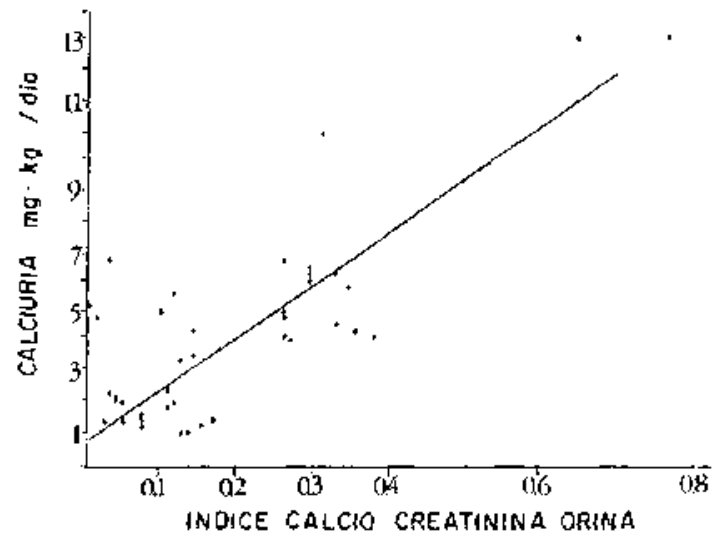

Figura 2: Relacion entre fndice calcio/creatinina en orina aisiada en ayunas ys. calcio en orina de $24 \mathrm{~h}$.

prueba de restricción simple de calcio este porcentaje disminuyó a 7\% global; sin embargo, si consideramos por separado a los treinta $y$ tres pacientes evaluados por restricción y sobrecarga, el diagnóstico no fue posible en $18 \%$ de los casos estudiados por este mérodo.

La subclasificación de los enfermos a través de estos ensayos se basa en la observación del margen de variación que experimenta el índice inicial al repetir su determinación bajo distintas condiciones. La variación experimentada por el indice calcio/creatinina entre la determinacion basal y la del ténnino de la prueba mostró una diferencia similar y estadísticamentc significativa en ambos tipos de hipercalciuria (renal y absortiva) cuando se aplicó la restricción y sobrecarga de calcio. En contraste, cuando se usó la restricción simple, el cambio experimentado por el índice en las formas absortivas fue también estadísticamente significativo, no así la variación ocurrida en las formas renales, que Io hicieron en un estrecho margen, sin significación estadística, lo que otorga a este método una sensibilidad que no es evidente en el anterior (figura 3).

\section{Comentario}

En esta evaluación de la hipercalciuria idiopática hemos intentado contestar algunas interrogantes no estudiadas previamente en nuestra población infantil y que pueden servir de referencia para futuros estudios. Hemos observado que en nifos sanos la mediana del índice calcio/creatinina en orina es baja e influida, en forma débil pero estadísticamente significativa, por la cxcreción

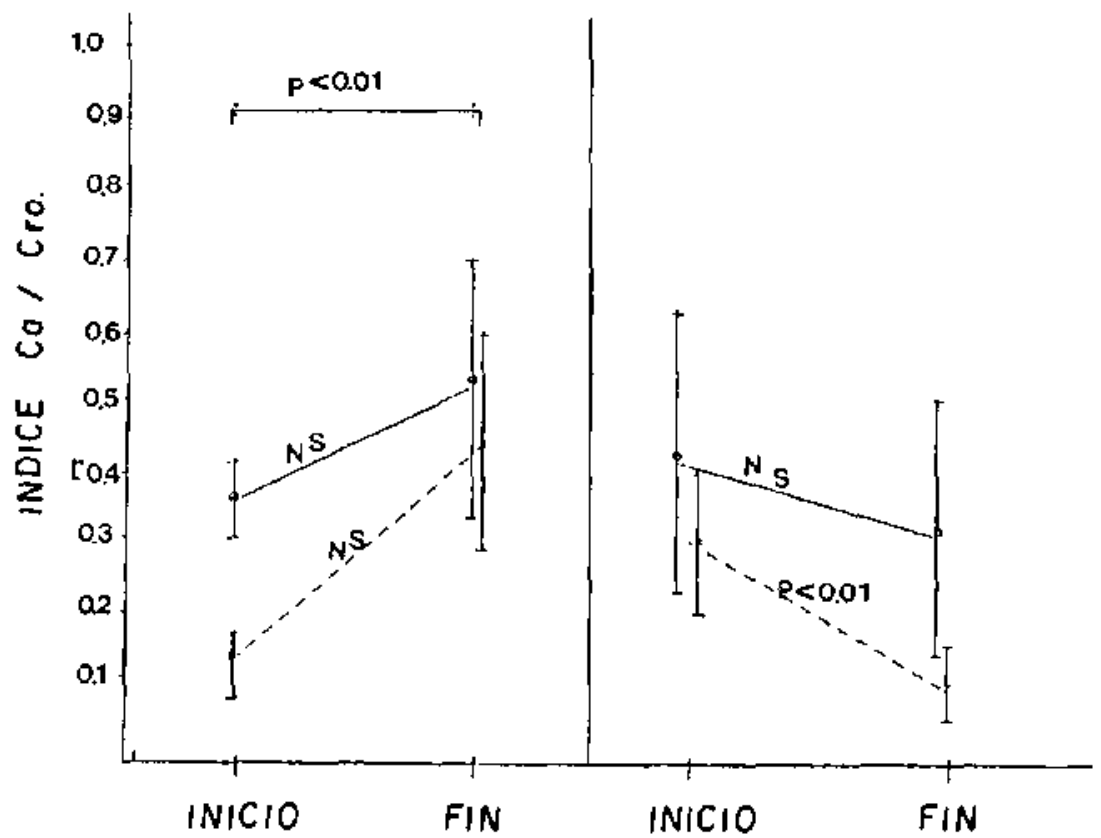

Figura 3: Sensibilidad del test de restricción y sobrecarga de calcio frente al tesı de restricción simple de calcio en el diagnostico diferencial de ha hipercalciuria idiopática. 
de sodio en orina. Ello puede explicar la amplia dispersión en los valores finales del índice calcio/ creatinina medido en condiciones basales. La relación entre natriuria y excreción de calcio en orina se estudió en sujetos normales, de donde se pudiera conjeturar que la asociación podría ser de mayor significación en pacientes con hipercalciuria.

Creemos igualmente que, dadas las marcadas difercncias en el medio ambiente y alimentación que se observa en distintas regiones del país, es necesario estudiar muestras locales, aunque los resulıados obtenidos sugiercn que cl rango ofrecido en la literatura intemacional ${ }^{4,21}$ es suficientemente amplio para acoger las variaciones que pudieran generarse en distintas poblaciones.

Se confirmó la validez del índice calcio/creatinina en orina aislada en ayunas, en reemplazo de la calciuria en orina de 24 horas, en el estudio clínico de la hiperealciuria idiopatica. La comodidad de la muestra única aislada de orina y la sencillez de las determinaciones requeridas ponen el procedimiento al alcance de los pediatras en la mayoría de las regiones de nuestro país.

A pesar que los ensayos aplicados en muestro estudio al diagnóstico diferencial de la hipercalciuria idiopática son objeto de controversia, se usan ampliamente debido a que permiten orientar una conducta terapéutica en la mayoria de los casos. Al compararlos observamos que las formas renal y absortiva se comportan estadísticamente en forma similar en la prueba de restricción y sobrecarga de $\mathrm{Ca}$, en tanto que la restricción simple de calcio y sodio genera diferencias significativas entre ambos subtipos, ya que sólo la forma absortiva cambia sus valores en forma estadísticamente significativa. Ello puede interpretarse con mejor capacidad de la prueba de restricción simple para diferenciar los subtipos de hipercalciuria, lo que es confinmado por la diferencia de resultados no concluyentes entre los dos ensayos.

La mayoría de nuestros pacientes correspondieron al subtipo absortivo, que es de difícil tratamiento debido a que el niño generalmente transgrede las indicaciones terapéuticas. Estas han sido analizadas en detalle en otras publicaciones $^{2,4}$. La influencia de factores dietéticos distintos al calcio sobre la calciuria, como el sodio y las proteínas, dificulta en gran medida el buen control de estos pacientes. Es probable que la hipercalciuria idiopática agrupe a dos tipos de pacientes, uno de origen metabólico-secundario a trastomo de la absorción intestinal o la excreción renal de calcio- y otro en que la afección es de origen dictético, con resultados erráticos en las pruebas y el efecto obtenido con diferentes tcrapéuticas.

Aclualmeate se considera que esta enfermedad pudiera no ser tan inocua como se pensaba antes. La constatación de litiasis al cabo de unos años de seguimiento en los pacientes que consultaron por hematuria, indica la conveniencia de su pesquisa oportuna y adecuado manejo cn pediatría. Publicaciones recientes ban destacado la existencia de altcraciones en el metabolismo óseo de estos enfermos, con disminución de hasta $10 \%$ de la densidad ósca frente a controles normales. En un estudio preliminar de las concentraciones séricas de hormona paratiroidea en nuestros pacientes, nos ha llamado la atención el estado de supresión en que se encuentra esta homona. Sin duda se han logrado avances en el dajagnóstico y tratamicnto de los ninfos portadores de este trastomo, con positiva repercusión en el estudio de las hematurias y litiasis nefrourológicas, pero todavía quedan muchas interrogantes por responder.

\section{Resumen}

El estudio de la hipercalciuria idiopática en pediatría requiere contar con información local. Se presentan los resultados de la medición de cxcreción urinaria de calcio en 97 niños sanos, medido a través del índice calciuria creatininuria, resultando un promedio de 0,13 y una mediana de 0,08 . Se evalúa igualmente la relación entre calcituria así determinada y la excreción urinaria de sodio ( $n: 47$ ), demostrándose una correlación estadísticamente significativa, $\mathrm{r}$ de $0,41, \mathrm{p}<0,01$. La validez del indice calcio/creatinina en orina aislada frente al calcio en orina de 24 horas (n: 44) se confirmó al obtener una alta correlación (r: $0,89, p<0,001$ ) entrambos. Al comparar la eficacia de la prueba de restricción y sobrecarga de calcio con la de restricción simple de calcio en el diagnostico diferencial de las hipercalciurias idiopáticas (n: 78) absortiva y renal, se observó que la exactitud del ensayo de restricción simple es mayor.

(Palabras clave: trastornos metabólicos del calcio, hipercalciuria idiopática, absortiva, renal, índice calcio/creatinina en orina.) 


\section{Referencias}

I. Stapleton FB, Roy III Sh, Noe HN: Hypencalciuria in Children with Hematuria. N Engl J Med 1984; 310 : $1345 \cdot 1348$.

2. CanofJ: Hipercalciuria Idiopática en Pediatría. Puesta al día. Pediatría al día en prensza.

3. Flocks $R$ h: Calcium exrction in the urine of Patients with renal or ureteral calculi. JAMA 1939; 113: 1466 1468.

4. Roy IIISh, Stapieton FB, Noe HN, Jerkins J: Hematuria Preceding Renal Calculus formation in children with Hypercalciuria. J Pediatr 1981; 99: 712-715.

5. Stapleton FB: Hypercalciuria in Children with Hematuria: an update. In: Strauss J; Growth, Immunossupression and Renal Disorders in Children, 1989. University of Miami Press, Coral Gables, Florids.
6. Proessmon W, Favere $N$, Goos $L$ : Urinary Calcium Creatinine Ratio in Healthy Newborns Symposium. Ninth Congress of the International Pediatric Nephrology Association, Israel, 1992.

7. Stapteton FB, Mac Kay Ch, Noe HN: Urotithiasis in Chitdren: The Role of Hypercalciuria. Pediatr Am, 1987: $10 ; 980-982$.

8. Heiliczer JD, Cononigo BB, Bishoff NP, Moore ES: Noncalculi Urinary Tract Disorders secondary to Idiopathic Hypercalciuria. Pediatr Clin North Am 1987; 34: 711-718.

9. Goldfard $S$ : Dietary Factors in the Pathogenesis and Prophylaxis of Nephrolithiasis Kidney Int 1988; 34: 544-555.

10. Cano FJ, Rodriguez $E$, Delucchi $A$, Wolff E: Hipercalciuria Idiopática. Diagnóstico Diferencial. Rev Chil Pediarr 1990; 61: 202-205

11. Stapleion FB: Ask the Expen, Pediatr Nephrol 1990; 4: $29-31$. 